\title{
Radiation Chemistry of Advanced TALSPEAK Flowsheet
}

\section{Fuel Cycle Research \& Development}

Prepared for

U.S. Department of Energy

Fuel Cycle Research and Development Separation and Waste Form Campaign

Bruce J. Mincher, Dean R. Peterman, Rocklan G. McDowell, Lonnie G. Olson Idaho National Laboratory Gregg Lumetta, Pacific Northwest National Laboratory August 28, 2013 FCR\&D-SEPA-2013-000295

PNNL-22712 


\section{DISCLAIMER}

This information was prepared as an account of work sponsored by an agency of the U.S. Government. Neither the U.S. Government nor any agency thereof, nor any of their employees, makes any warranty, expressed or implied, or assumes any legal liability or responsibility for the accuracy, completeness, or usefulness, of any information, apparatus, product, or process disclosed, or represents that its use would not infringe privately owned rights. References herein to any specific commercial product, process, or service by trade name, trade mark, manufacturer, or otherwise, does not necessarily constitute or imply its endorsement, recommendation, or favoring by the U.S. Government or any agency thereof. The views and opinions of authors expressed herein do not necessarily state or reflect those of the U.S. Government or any agency thereof. 


\section{ABSTRACT}

This report summarizes the results of initial experiments designed to understand the radiation chemistry of an Advanced TALSPEAK process for separating trivalent lanthanides form the actinides. Biphasic aerated samples were irradiated and then analyzed for post-irradiation constituent concentrations and solvent extraction distribution ratios. The effects of irradiation on the TALSPEAK and Advanced TALSPEAK solvents were similar, with very little degradation of the organic phase extractant. Decomposition products were detected, with a major product in common for both solvents. This product may be responsible for the slight increase in distribution ratios for Eu and Am with absorbed dose, however; separation factors were not greatly affected. 


\section{CONTENTS}

ABSTRACT iii

ACRONYMS vii

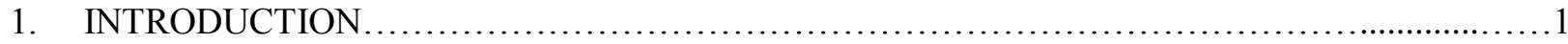

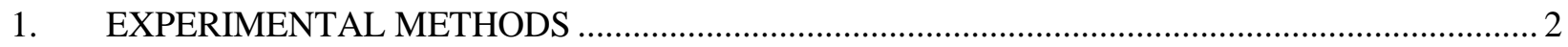

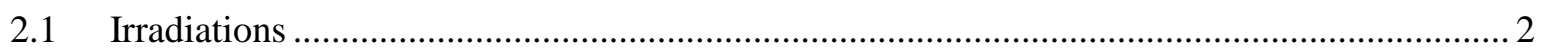

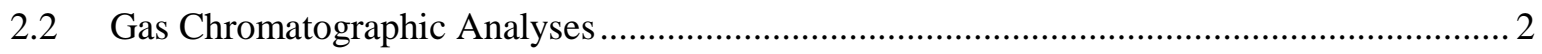

2.3 Ion Chromatographic Analyses ................................................................................ 2

2.4 High performance Liquid Chromatogarphic Analysis ......................................................... 2

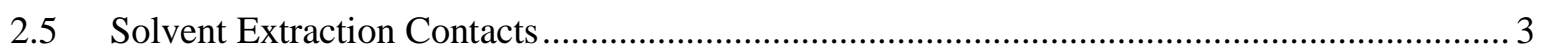

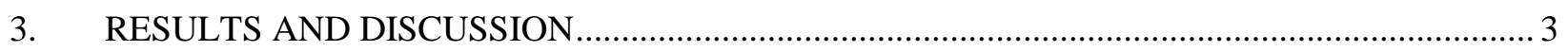

3.1 HEH[EHP] Radiation Chemistry............................................................................. 3

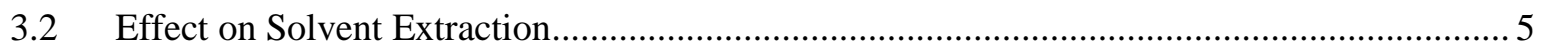

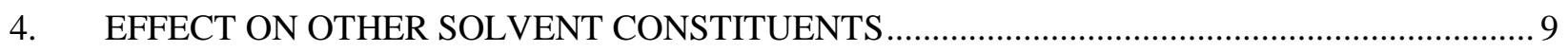

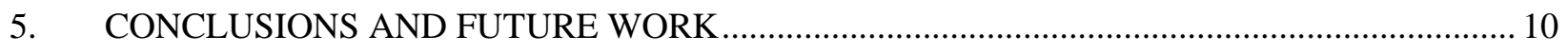

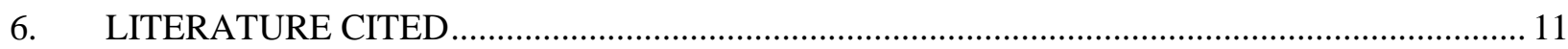

\section{FIGURES}

Figure 1 The change in concentration for HEH[EHP] (boxes) or HDEHP (diamonds) when irradiated as components of either the Advanced TALPEAK or TALSPEAK process solvents, in contact with the same aqueous phase with air-sparging. Error bars shown are $\pm 5 \%$ based on replicate analyses. See text for

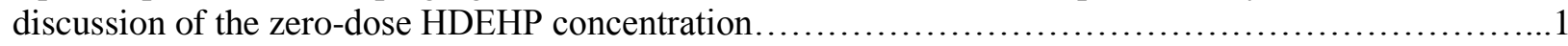

Figure 2 The increase in peak area with absorbed dose of radiolysis-product species for $\gamma$-irradiated samples of HEH[EHP] or HDEHP when irradiated as components of either the Advanced TALPEAK or TALSPEAK process solvents, in contact with the same aqueous phase with air-sparging.................5

Figure 3 The solvent extraction of Am and Eu with 1.0 M HEH[EHP]/dodecane from $\mathrm{pH} 3.5,0.05 \mathrm{M}$ DTPA/1.5 M lactic acid aqueous phase. Error bars shown are $\pm 10 \%$. Both phases were irradiated in contact with each other with air-sparging.....

Figure 4 The solvent extraction of Am and Eu with 0.5 M HDEHP/dodecane from $\mathrm{pH} 3.5,0.05 \mathrm{M}$ DTPA/1.5 M lactic acid aqueous phase. Error bars shown are $\pm 10 \%$. Both phases were irradiated in contact with each other with air-sparging.

Figure $5 \mathrm{Am}$ and Eu distribution ratios as a function of absorbed dose for a) the HDEHP solvent and a fresh lactic acid aqueous phase b) the HEH[EHP] solvent and a fresh citric acid aqueous phase. 
Figure 6 The radiolytic decomposition of aqueous lactic acid in the aerated, irradiated biphasic system with an organic phase composed of $0.5 \mathrm{M}$ HDEHP/dodecane (closed diamonds: TALSPEAK) or $1.0 \mathrm{M}$ $\mathrm{HEH}[\mathrm{EHP}] /$ dodecane (open squares: Advanced TALSPEAK) . Error bars shown are $\pm 3 \%$ based on repetitive analyses.

Figure 7 The radiolytic decomposition of aqueous DTPA in the aerated, irradiated biphasic system with an organic phase composed of $0.5 \mathrm{M}$ HDEHP/dodecane (open diamonds: TALSPEAK) or $1.0 \mathrm{M}$ $\mathrm{HEH}[\mathrm{EHP}] /$ dodecane (closed triangles: Advanced TALSPEAK) . Error bars shown are $\pm 3 \%$ based on

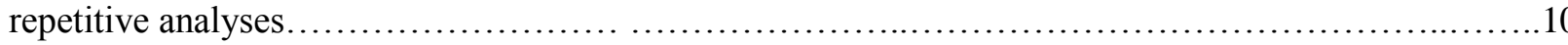




\section{ACRONYMS}

$\begin{array}{ll}\text { ALSEP } & \text { Actinide Lanthanide SEParations process } \\ \text { DTPA } & \text { Diethylenetriaminepentaacetic acid } \\ \text { FCR\&D } & \text { Fuel Cycle Research and Development } \\ \text { GC-FID } & \text { Gas chromatography flame ionization detection } \\ \text { HDEHP } & \text { Diethylhexylphosphoric acid } \\ \text { HEDTA } & \text { 2-hydroxethylethylenediaminetriacetic acid } \\ \text { HEH[EHP] } & \text { 2-ethylhexylphosphonic acid mono-2-ethylhexyl ester } \\ \text { H}_{2} \text { MEHP } & \text { Monoethylhexylphosphoric acid } \\ \text { HPLC } & \text { High Performance Liquid Chromatography } \\ \text { INL } & \text { Idaho National Laboratory } \\ \text { PNNL } & \text { Pacific Northwest National Laboratory }\end{array}$

TALSPEAK Trivalent Actinide Lanthanide Separation by Phosphorous reagent Extraction from Aqueous Komplexes

TRUEX Transuranic Extraction Process 



\section{SEPARATIONS AND WASTE FORMS}

\section{INTRODUCTION}

Separating the minor actinide elements (americium and curium) from the fission product lanthanides is an important step in closing the nuclear fuel cycle. Isolating the minor actinides will allow transmuting them to short lived or stable isotopes in fast reactors, thereby reducing the long-term hazard associated with these elements. ${ }^{[1-2]}$ A two-step method has been adopted by the Fuel Cycle Research and Development (FCR\&D) program to separate the minor actinides from acidic high-level waste. The first step involves co-extracting the lanthanides and minor actinides using the transuranic extraction (TRUEX) process. ${ }^{[3]}$ The second step uses the Trivalent Actinide-Lanthanide Separations by Phosphorus-reagent Extraction from Aqueous Complexes (TALSPEAK) process to separate the minor actinides from the lanthanides. ${ }^{[4-5]}$ The tandem TRUEX-TALSPEAK approach has been demonstrated on irradiated fuel at a laboratory scale. ${ }^{[6]}$ However, the TALSPEAK process suffers the disadvantage of slow extraction kinetics and high sensitivity to the aqueous phase $\mathrm{pH}$. To overcome these disadvantages, a variation of the TALSPEAK process has been proposed which replaces the traditionally used bis(2-ethylhexyl)phosphoric acid (HDEHP) extractant with 2-ethylhexylphosphonic acid mono-2-ethylhexyl ester (HEH[EHP]). ${ }^{[7]}$ This is referred to as an Advanced TALSPEAK process. The key advantages of switching to the HEH[EHP] extractant are reported to be: less dependence of the process performance on the aqueous solution $\mathrm{pH}$, more predictable extraction behavior, and more rapid extraction kinetics. The Advanced TALSPEAK process is being considered as a candidate to replace conventional TALSPEAK in the FCR\&D full recycle case study.

In addition to the Advanced TALSPEAK process, researchers at Argonne National Laboratory (ANL) and Pacific Northwest National Laboratory (PNNL) are investigating new solvent formulations that combine the functional steps achieved by the TRUEX and TALSPEAK processes into a single solvent extraction cycle. This approach has led to the development of two processes that involve combining a bifunctional neutral extractant with an acidic extractant. ${ }^{[8,9]}$ The neutral extractant serves to co-extract the trivalent actinides and lanthanides from $\mathrm{HNO}_{3}$ solutions (much like the TRUEX process), while the acidic extractant serves to hold the trivalent lanthanides in the organic phase while the actinides are selectively stripped into a carboxylate-buffered solution containing a polyaminocarboxylate ligand (a ReverseTALSPEAK type of process). ${ }^{[8-9]}$ One such approach is the Actinide Lanthanide SEParations (ALSEP) process which also utilizes HEH[EHP] as the acidic extractant. This new complexing agent varies from conventional HDEHP in that one of the diethylhexyl groups is bonded directly to the phosphorous atom, rather than through an ether linkage. Since ether linkages are well-known to be susceptible to rupture during radiolysis, the radiation chemistry of $\mathrm{HEH}[\mathrm{EHP}]$ is of interest and understanding its radiolytic degradation is relevant to both the Advanced TALSPEAK process and the ALSEP processes. A collaborative study of the radiolysis of HEH[EHP] and the Advanced TALSPEAK formulation was therefore initiated in FY13 by INL and PNNL. This report describes those results and is written in completion of milestone M3FT-IN0302071, Level 3, the goal of which was to develop detailed scope of lab-scale testing, perform testing and to issue this report. 


\section{EXPERIMENTAL METHODS}

\section{$2.1 \quad$ Irradiations}

Samples of the Advanced TALSPEAK solvent (1.0 M HEH[EHP] /dodecane) were batch irradiated in contact with an equal volume of $0.05 \mathrm{M}$ DTPA/1.5 M lactate aqueous phase, adjusted to $\mathrm{pH} 3.5$ with either nitric acid or ammonium hydroxide, as necessary. For comparison, the conventional TALSPEAK organic phase (0.5 M HDEHP/dodecane) was also irradiated in contact with an equal volume of the same aqueous phase. The samples were air-sparged during irradiation to best simulate process conditions. The gamma-source is a Nordion GammaCell 220 Excel ${ }^{60} \mathrm{Co}$ gamma irradiator. The center-line gamma dose rate in the sample chamber was $\sim 5.7 \mathrm{kGy} / \mathrm{hr}$ at the time of these experiments, based on decay-corrected Fricke dosimetry.

\subsection{Gas Chromatographic Analyses}

The presence of acidic functional groups on HDEHP and HEH[EHP] and their possible degradation products required that these compounds be derivatized prior to analysis by gas chromatography flame ionization detection (GC-FID). The method used for derivatization was adapted from that previously used for the analysis of dibutylphosphoric acid. ${ }^{[10]}$ Samples were diluted with hexane and derivatized with 300 $\mu \mathrm{L}$ of $\sim 0.3 \mathrm{~mol} / \mathrm{L}$ diazomethane in hexane. This produced the methyl ester of the phosphoric acid functional groups in the target compounds. The samples were then analyzed using a Thermo Scientific Trace ULTRA GC using a Thermo Scientific TG-35MS capillary column (30m x $0.32 \mathrm{~mm}$ ID x $0.5 \mu \mathrm{m}$ film). The He carrier gas flow was $2.0 \mathrm{~mL} / \mathrm{min}$ with an $80 \mathrm{~mL} / \mathrm{min}$ split flow. Oven operating conditions started with a $2 \mathrm{~min}$ hold at $70^{\circ} \mathrm{C}$, followed by a ramp at $20^{\circ} \mathrm{C} / \mathrm{min}$ to $240^{\circ} \mathrm{C}$ then $40^{\circ} \mathrm{C} / \mathrm{min}$ to $280^{\circ} \mathrm{C}$, and finished with an $8.25 \mathrm{~min}$ hold at $280^{\circ} \mathrm{C}$. A Thermo AS3000 auto sampler was used for all injections, employing a $1 \mu \mathrm{L}$ hot injection with the inlet set at $250{ }^{\circ} \mathrm{C}$ and 5 second pre-injection dwell time. The FID was held constant at $250^{\circ} \mathrm{C}$. The fuel gas for the FID was a mixture of $350 \mathrm{~mL} / \mathrm{min}$ air and 35 $\mathrm{mL} / \mathrm{min}$ hydrogen with $30 \mathrm{~mL} / \mathrm{min}$ nitrogen as a makeup gas.

\subsection{Ion Chromatographic Analyses}

A Dionex ICS-5000 ion chromatograph with eluent generator, autosampler, gradient pump with degasser, conductivity detector, anion self-regenerating suppressor, continuously regenerated anion trap column, and ASRS 300 anion suppressor was used with an IonPac AS-11HC (2mm x $250 \mathrm{~mm})$ analytical column and AG-11HC (2mm x 50mm) guard column. The flow rate was isocratic at $0.38 \mathrm{~mL} / \mathrm{min}$. The step gradient was maintained using an EluGen II KOH cartridge and eluent generator. The eluent gradient was $3.0 \mathrm{mmol} / \mathrm{L} \mathrm{KOH}$ for 10 minutes followed by an $80.0 \mathrm{mmol} / \mathrm{L}$ flush for $5 \mathrm{~min}$ and a $5 \mathrm{~min}$ re-equilibration time at $3.0 \mathrm{mmol} / \mathrm{L}$.

\subsection{High Performance Liquid Chromatographic Analysis}

The DTPA concentration was determined using an High Performance Liquid Chromatography ( HPLC) system consisting of a Waters Model 717 Autosampler with a Model 600e Multisolvent Delivery Pump System and Model 2996 photodiode array detector. The separation was performed using a SIELC 4.6x250 mm PrimeSep D column with $5 \mu \mathrm{m}$ particles and $100 \AA$ pores using a gradient consisting of $100 \%$ solvent A to $100 \%$ solvent B over 12 min, ramp back to $100 \%$ A over 1 minute and holding for 7 minutes. An additional 6 minutes for re-equilibration was allowed between sample injections. Solvent A 
was $10 \%$ acetonitrile/90\% water with the addition of $2 \mu \mathrm{L} / \mathrm{L}$ concentrated sulfuric acid and $0.02 \%(w / w)$ $\mathrm{CuSO}_{4}$. Solvent B was $50 \%$ acetonitrile $/ 50 \%$ water with the addition of $20 \mu \mathrm{L} / \mathrm{L}$ concentrated sulfuric acid and $0.02 \%(\mathrm{w} / \mathrm{w}) \mathrm{CuSO}_{4}$.

\subsection{Solvent Extraction Contacts}

Solvent extraction experiments were performed at PNNL using the irradiated organic phase from the above, but with extraction from fresh aqueous phases based on lactic acid for the HDEHP organic, or citric acid for the HEH[EHP] organic. This allowed for comparison of the two processes from the aqueous phases proposed for their process conditions. A lactic acid stock solution consisting of $0.001 \mathrm{M}$ $\mathrm{EuCl}_{3}+0.05 \mathrm{M}$ DTPA + 1.5 M lactic acid at $\mathrm{pH} 3.5$ was prepared by mixing $0.25 \mathrm{~mL} 0.1 \mathrm{M} \mathrm{EuCl}_{3}, 1.25$ $\mathrm{mL} 1 \mathrm{M}$ DTPA, and $12.058 \mathrm{~mL} 3.11 \mathrm{M}$ lactic acid; adjusting to $\mathrm{pH} \sim 3.4$ with $19 \mathrm{M} \mathrm{NaOH}$; then diluting to $25 \mathrm{~mL}$ with deionized water. The $\mathrm{pH}$ of the resulting solution was 3.49. In a similar way, a citric acid stock consisting of $0.001 \mathrm{M} \mathrm{EuCl}_{3}+0.125 \mathrm{M}$ HEDTA $+0.2 \mathrm{M}$ citric acid at $\mathrm{pH} 3.0$ was prepared by mixing $0.25 \mathrm{~mL} 0.1 \mathrm{M} \mathrm{EuCl}_{3}, 2.86 \mathrm{~mL} 1.093 \mathrm{M}$ HEDTA, $2.0 \mathrm{~mL} 2.5 \mathrm{M}$ citric acid and $15 \mathrm{~mL}$ deionized water; adjusting to $\mathrm{pH} \sim 2.9$ with $19 \mathrm{M} \mathrm{NaOH}$; then diluting to $25 \mathrm{~mL}$ with deionized water. The $\mathrm{pH}$ of the resulting solution was 2.99 .

Each of the irradiated HDEHP samples was then contacted with an equal volume of the $0.001 \mathrm{M} \mathrm{EuCl}_{3}+$ $0.05 \mathrm{M}$ DTPA + 1.5 M lactic acid/pH 3.5 solution spiked with ${ }^{241} \mathrm{Am}$ and ${ }^{155}$ Eu tracers. Similarly, the irradiated HEH[EHP] solvents were contacted with equal volumes of the $0.001 \mathrm{M} \mathrm{EuCl}_{3}++0.125 \mathrm{M}$ HEDTA + 0.2 M citric acid/pH 3.0 solution spiked with ${ }^{241} \mathrm{Am}$ and ${ }^{155} \mathrm{Eu}$ tracers. Each contact was performed by vortex mixing at $1900 \mathrm{rpm}$ for $3 \mathrm{~h}$, centrifuging, and sampling for gamma analysis. The counts associated with ${ }^{241} \mathrm{Am}$ at $59.5 \mathrm{keV}$ were corrected for the contribution from the ${ }^{155} \mathrm{Eu}$ emission at $60 \mathrm{keV}$. In the case of the HEH[EHP] extraction contacts, interference from the $60 \mathrm{keV}{ }^{155} \mathrm{Eu}$ emission was too great to yield reliable ${ }^{241} \mathrm{Am}$ counting data. In this case, a separate run was performed in which only the ${ }^{241} \mathrm{Am}$ spike was included. The $\mathrm{pH}$ of each aqueous phase was determined after completion of the contact.

Solvent extraction experiments were also performed at INL for supplemental information, using the aqueous phase from the initial irradiations, following spiking of that aqueous phase with ${ }^{243} \mathrm{Am}$ and ${ }^{154} \mathrm{Eu}$. This allowed for comparison of irradiated HDEHP and HEH[EHP] radiolysis under otherwise identical conditions. The organic to aqueous phase volume ratio used for all contacts was $\mathrm{O} / \mathrm{A}=1.0$. The phases were mixed for two minutes using a vortex mixer and then centrifuged to separate. The solvent extraction experiments were performed at ambient temperature $\left(21 \pm 2{ }^{\circ} \mathrm{C}\right)$. The concentration of radiotracers present in the organic and aqueous phases was determined by gamma spectrometry. The distribution ratio, $D_{M}$, was calculated as the ratio of activity in the organic and aqueous phases, $D_{M}=[M]_{\text {org }} /[M]_{\text {aq }}$.

\section{RESULTS AND DISCUSSION}

\subsection{HEH[EHP] Radiation Chemistry}

The effect of $\gamma$-rays on the concentration of HEH[EHP] irradiated as a component of the aerated, biphasic Advanced TALSPEAK process is shown in Fig. 1. Also shown is the change in concentration for HEDHP as a function of absorbed dose for irradiation under identical conditions. In both cases there appears to be no concentration change due to irradiation. 


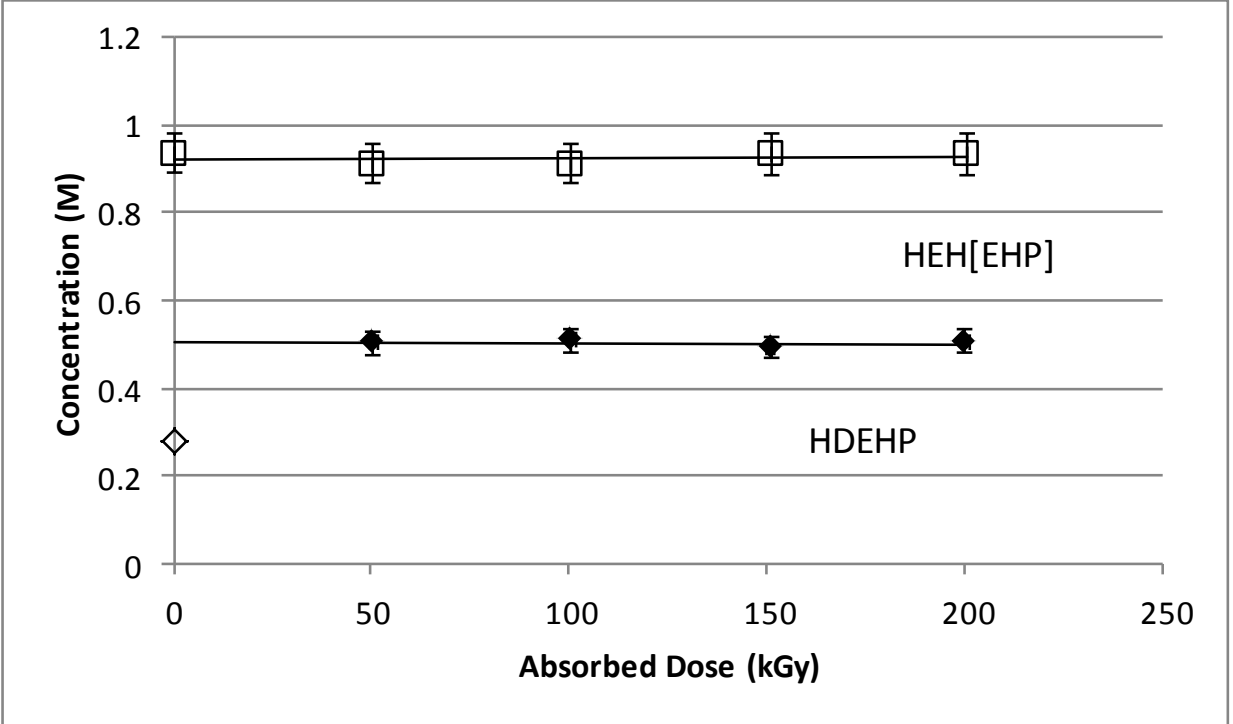

Figure 1. The change in concentration for HEH[EHP] (boxes) or HDEHP (diamonds) when irradiated as components of either the Advanced TALPEAK or TALSPEAK process solvents, in contact with the same aqueous phase with air-sparging. Error bars shown are $\pm 5 \%$ based on replicate analyses. See text for discussion of the zero-dose HDEHP concentration.

However, it should be noted that the initial, non-irradiated solution concentration of HDEHP (indicated with the open diamond in Fig. 1) was measured to be nearly a factor of two lower than the initially prepared $0.5 \mathrm{M}$ solution, and the same amount lower than the constant concentration measured in the irradiated samples. Although the cause of this has not been determined, it is believed that this is an artificially low result. If this is the case, to an absorbed dose as high as $\sim 200 \mathrm{kGy}$ there was no measurable decrease in the concentration of either compound due to irradiation, suggesting that both have adequate stability for fuel cycle applications. However, products analysis is also important, since small amounts of radiolysis products may be deleterious to system performance.

Although Fig. 1 showed no obvious change in the concentrations of the ligands, small amounts of products were detectable by GC-FID as peaks appearing at new retention times (RT). During the analyses of both the conventional TALSPEAK and Advanced TALSPEAK solvents a new peak was found $(\mathrm{RT}=$ $8.71 \mathrm{~min}$ ) in irradiated samples; indicating its origin in the radiolysis of both HDEHP and HEH[EHP]. This is shown in Fig. 2. Additionally, an unknown peak appeared in samples of the Advanced TALSPEAK solvent with RT $=9.61 \mathrm{~min}$, also shown in Fig. 2. This peak was present in unirradiated solution, but increased with absorbed dose. 


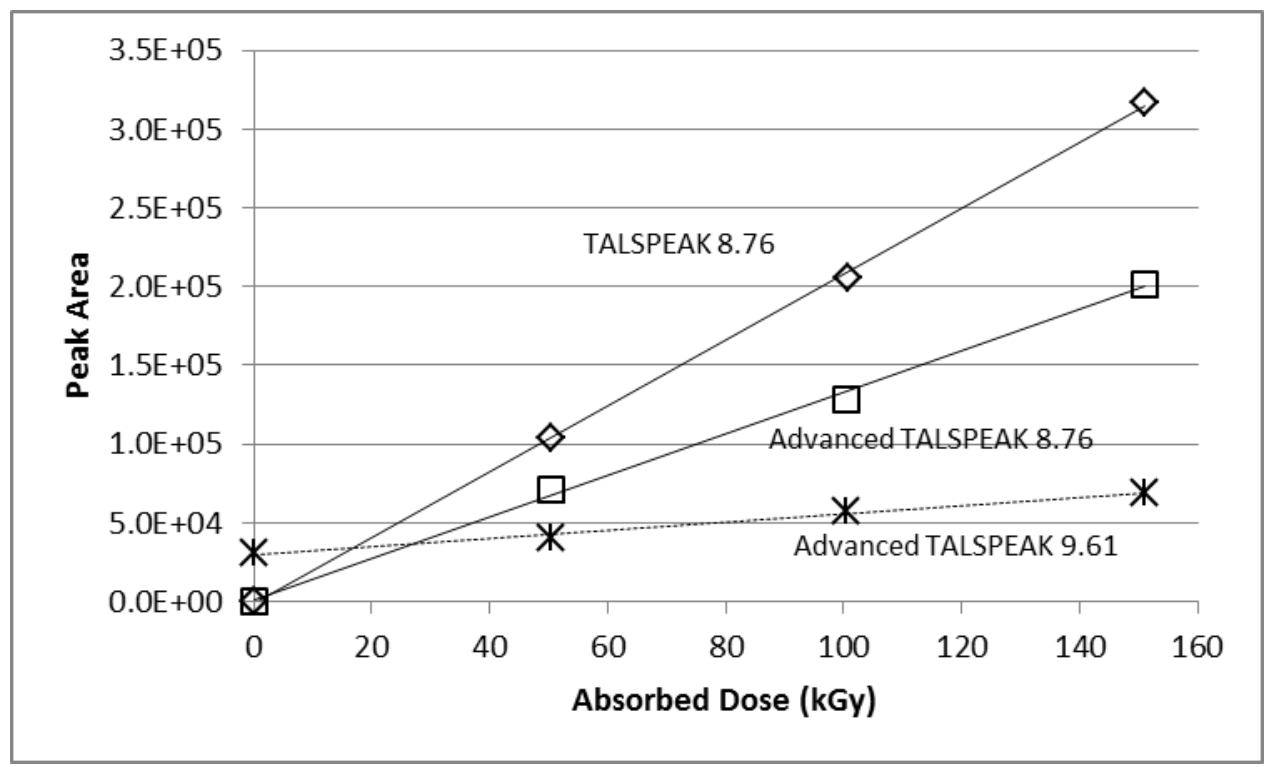

Figure 2. The increase in peak area with absorbed dose of radiolysis-product species for $\gamma$-irradiated samples of HEH[EHP] or HDEHP when irradiated as components of either the Advanced TALPEAK or TALSPEAK process solvents, in contact with the same aqueous phase with air-sparging.

The product of HDEHP irradiation is $\mathrm{H}_{2}$ MEHP, ${ }^{[11,12]}$ resulting from rupture of the ethylhexyl ether linkage and this is the most probable species corresponding to $\mathrm{RT}=8.76 \mathrm{~min}$. Ether linkages are readily susceptible to radiolytic rupture. Crown ethers, for example, are radiolyzed to non-cyclic products by ruptures at $\mathrm{C}-\mathrm{O}$ bonds, ${ }^{[13]}$ with decomposition initiated by formation of a carbon-centered radical adjacent to the ether oxygen ${ }^{[14]}$ due to either ${ }^{\circ} \mathrm{H}$ atom abstraction, ${ }^{[15]}$ as confirmed by fast rate constants for the reaction of ${ }^{\circ} \mathrm{H}$ atom and ${ }^{\circ} \mathrm{OH}$ radical with crown ethers, ${ }^{[16]}$ or by decay of the crown ether radical cation. ${ }^{[17]}$ That the 8.76 min product occurs at higher concentrations in the TALSPEAK formulation supports its production by rupture of the ether linkage, since HDEHP, although present at a lower initial concentration, has twice as many ether sites available as does HEH[EHP]. For the radiolysis of the latter species, $\mathrm{H}_{2} \mathrm{MEHP}$ can only be produced by dealkylation at the $\mathrm{C}-\mathrm{P}$ bond.

The product at $9.61 \mathrm{~min}$ RT is found only in Advanced TALSPEAK solutions, has a non-zero concentration in unirradiated samples and increased in concentration with absorbed dose. This suggests that it is an impurity associated with either synthesis or degradation of HEH[EHP] that is also produced by radiolytic degradation of that compound. The product may be monoethylhexylphosphonic acid, (or a degradation product of this species) which would be the product of the rupture of the single ether linkage in HEH[EHP].

\subsection{Effect on Solvent Extraction}

Samples of the TALSPEAK and Advanced TALSPEAK organic phases that were irradiated in the presence of the lactic acid aqueous phase were used in solvent extraction contacts. The results for the Advanced TALSPEAK contacts are shown in Fig. 3. Despite the nearly constant concentration of $\mathrm{HEH}[\mathrm{EHP}]$ in these irradiated samples it can be seen that the distribution ratios for both Am and Eu increased with absorbed dose; at a slightly faster rate for Am. The separation factor $\alpha_{\text {EuAm }}$ decreased moderately from $\sim 90-100$ at $0-50 \mathrm{kGy}$ to 74 at $200 \mathrm{kGy}$ absorbed dose. The moderately increasing distribution ratios shown may be attributed to the accumulation of $\mathrm{H}_{2} \mathrm{MEHP}$ and/or the proposed phosphonic acid radiolysis product. 


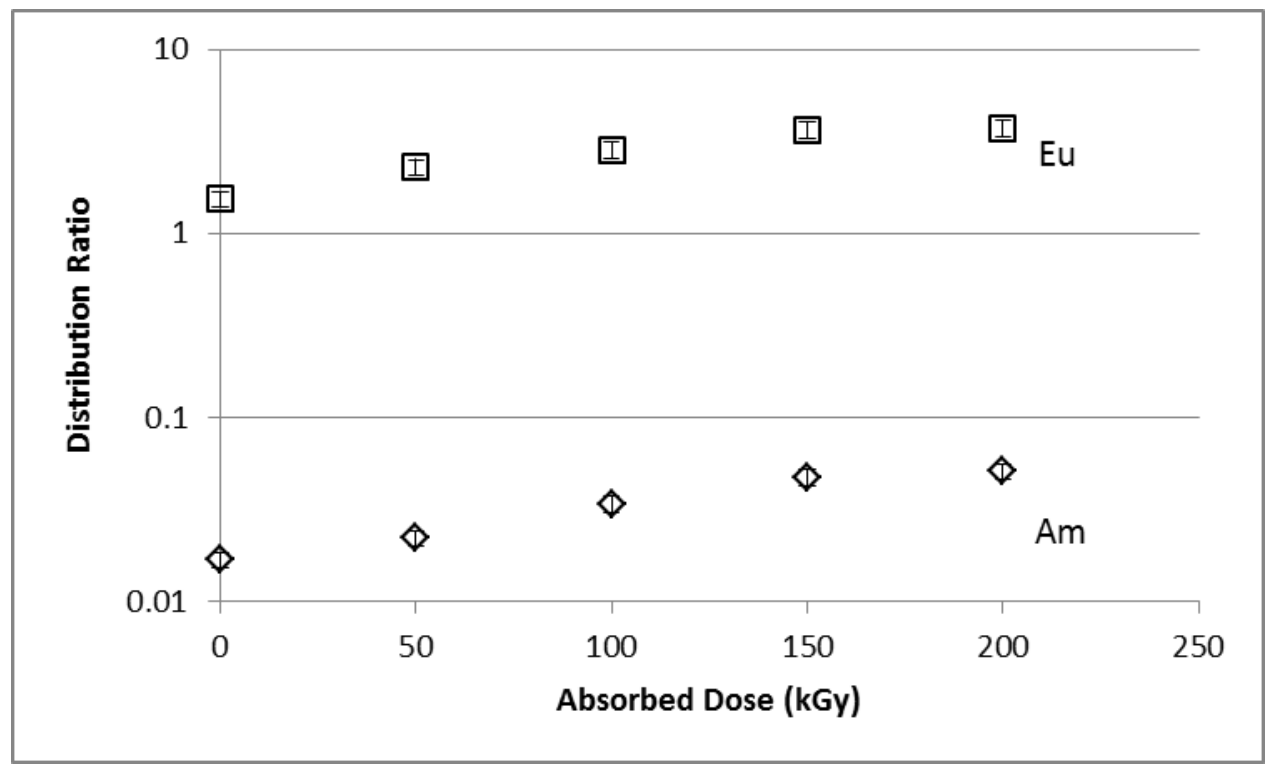

Figure 3. The solvent extraction of Am and Eu with 1.0 M HEH[EHP]/dodecane from $\mathrm{pH} 3.5,0.05 \mathrm{M}$ DTPA/1.5 M lactic acid irradiated aqueous phase. Error bars shown are $\pm 10 \%$. Both phases were irradiated in contact with each other with air-sparging.

The results for similar irradiations of conventional TALSPEAK followed by solvent extraction contacts are shown in Fig. 4. Similarly, there was an increase in the distribution ratios for both metals with absorbed dose at a similar rate for both metals, probably due to accumulation of $\mathrm{H}_{2} \mathrm{MEHP}$. Except for the initial, unirradiated sample, the $\alpha_{\text {EuAm }}$ remained constant at $\sim 50$. It can also be seen in Fig. 4 that the initial $D_{\mathrm{Eu}}$ and $D_{\mathrm{Am}}$ are lower than expected for the trend in these curves. This is regarded as further evidence that the concentration of the HDEHP in the unirradiated sample was anomalously low, consistent with the data shown in Fig. 1. Thus, for both HDEHP and HEH[EHP] organic phases a gradual increase in both actinide and lanthanide distribution ratios was found with respect to absorbed dose for samples irradiated in the presence of a the lactic acid buffered aqueous phase. This may be reasonably attributed to the likely degradation products proposed for each.

However, these results for the conventional TALSPEAK irradiations disagree with those measured previously, where it was reported that distribution ratios for both Am and Eu gradually decreased with absorbed dose for samples of $0.17 \mathrm{M} \mathrm{HDEHP/dodecane} \mathrm{irradiated} \mathrm{in} \mathrm{the} \mathrm{presence} \mathrm{of} \mathrm{a} \mathrm{lactic} \mathrm{acid/DTPA}$ aqueous phase; ${ }^{[18]}$ the only difference being that the samples were not aerated during irradiation in the previous work. However, the same $\mathrm{H}_{2} \mathrm{MEHP}$ product would be expected under reducing conditions since dissociative electron attachment would also rupture the C-O bond. ${ }^{[19]}$ Therefore, the difference may be due to the ratio of produced HDEHP to $\mathrm{H}_{2} \mathrm{MEHP}$, rather than aeration. For example, Tachimori reported that a maximum $D_{\mathrm{Am}}$ was obtained at a mole ratio of 2:1 HDEHP: $\mathrm{H}_{2}$ MEHP. ${ }^{[12]}$ 


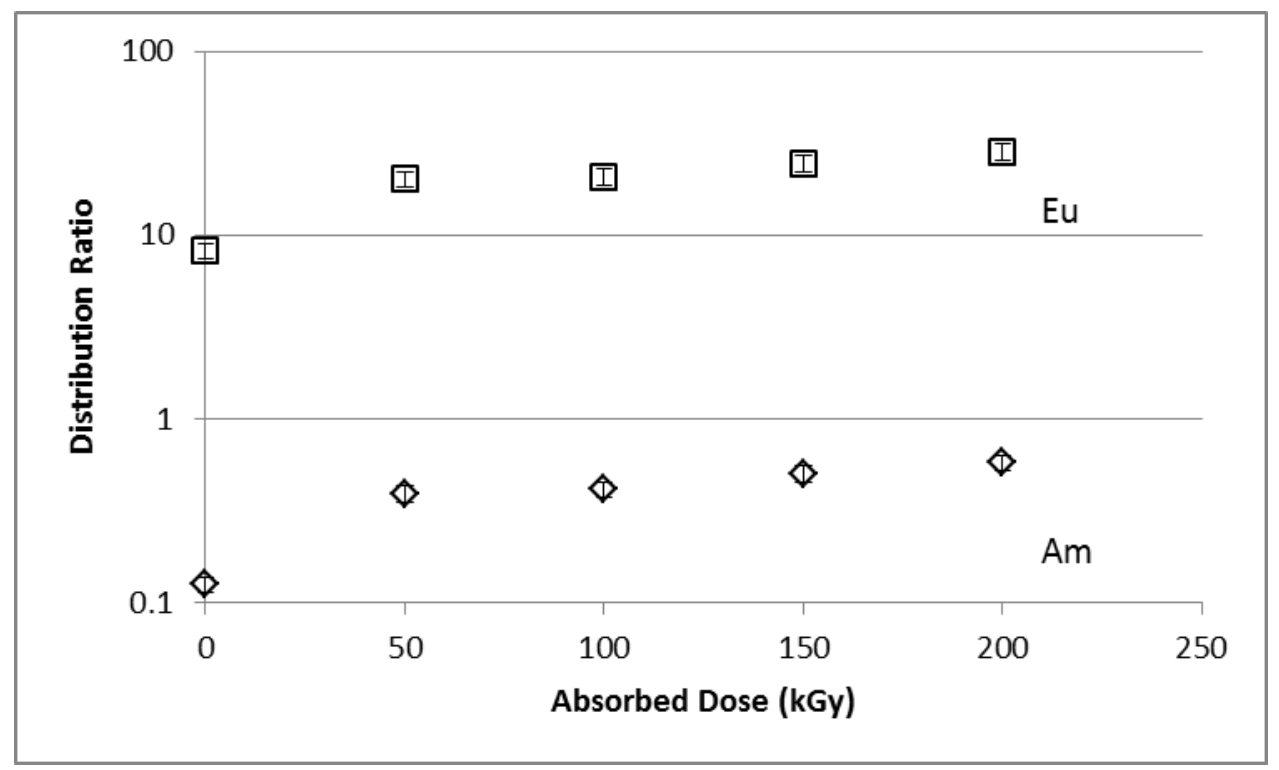

Figure 4. The solvent extraction of Am and Eu with 0.5 M HDEHP/dodecane from $\mathrm{pH} 3.5,0.05 \mathrm{M}$ DTPA/1.5 M lactic acid irradiated aqueous phase. Error bars shown are $\pm 10 \%$. Both phases were irradiated in contact with each other with air-sparging.

These irradiated organic phases were also used in solvent extraction experiments with fresh aqueous phases. For TALSPEAK, a fresh lactic acid aqueous phase was used, while for Advanced TALSPEAK a citric acid phase was substituted for the lactic acid solution, as described in the Experimental Section 2.3. 

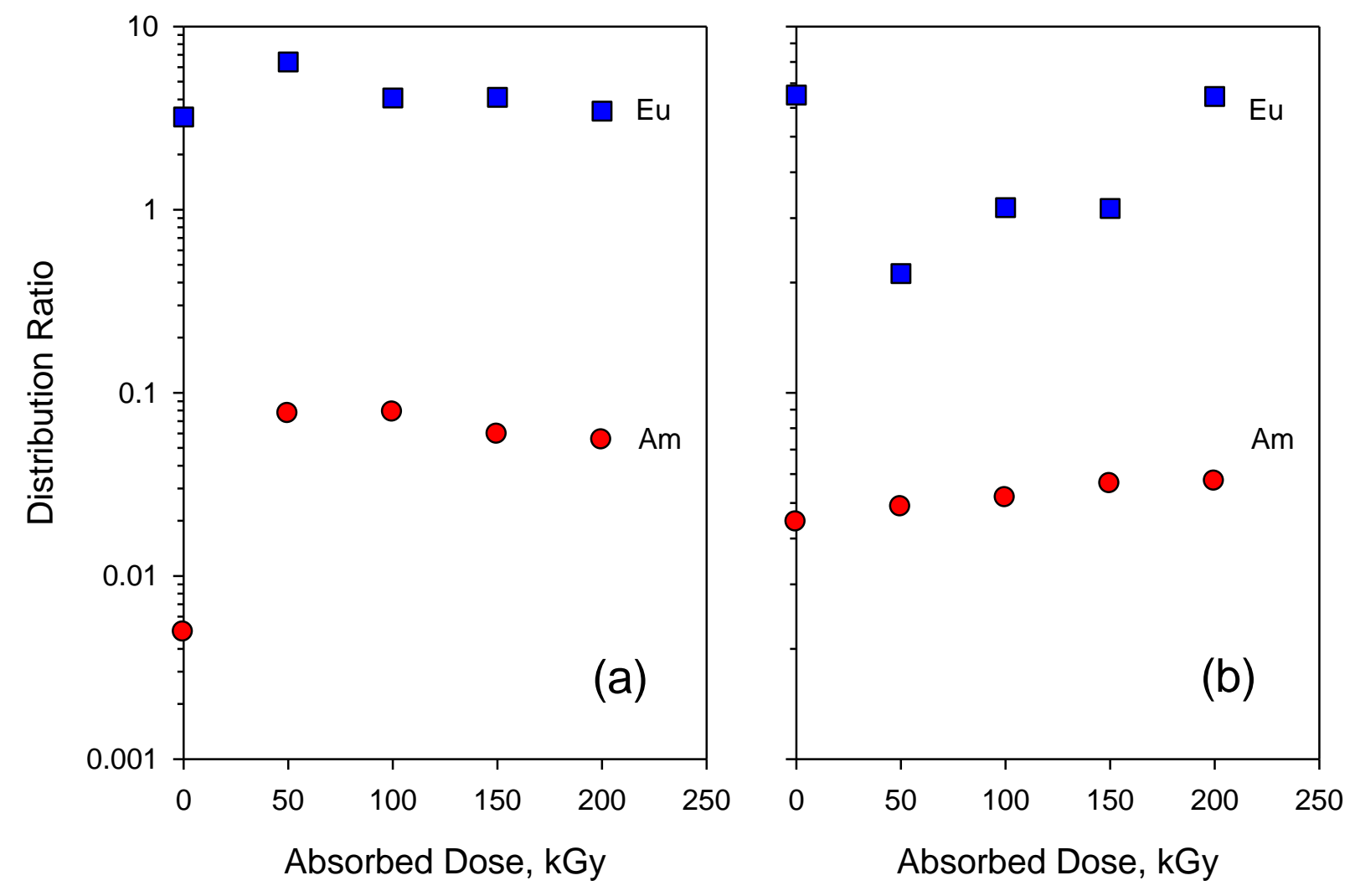

Figure 5. Am and Eu distribution ratios as a function of absorbed dose for a) the HDEHP solvent and a fresh lactic acid aqueous phase b) the HEH[EHP] solvent and a fresh citric acid aqueous phase.

Fig. 5a presents the Am and Eu distribution ratios as a function of absorbed dose for the same irradiated conventional TALSPEAK solvent for extractions from a fresh aqueous phase. For this HDEHPcontaining solvent, there is an apparent initial increase in the $D_{\mathrm{Am}}$ value upon irradiation to $50 \mathrm{kGy}$, with the $D_{\text {Am }}$ remaining nearly constant to slightly decreasing at still higher absorbed doses. A similar but less dramatic trend was found for Eu $D$ values. Once again, this is attributed to the unirradiated TALSPEAK sample having an anomalously low HDEHP concentration (see also Figs. 1 and 4). Thus, it is assumed that the distribution ratios here were unaffected by absorbed $\gamma$-dose. Since the distribution ratios for this solvent increased slightly following irradiation in contact with essentially the same aqueous phase (Fig. 4) this may indicate that the presumed $\mathrm{H}_{2} \mathrm{MEHP}$ acid product responsible for rising distribution ratios may have partially partitioned to the fresh aqueous phase to generate the data seen in Fig. 5a.

In the case of the Advanced TALSPEAK HEH[EHP]-containing solvent shown in Fig. $5 \mathrm{~b}$, the $D_{\text {Am }}$ values for extraction from a fresh citric acid aqueous phase show a gradual increase, similar to that found for extraction into the lactic acid aqueous phase in Fig. 4. The Eu data are more scattered for the HEH[EHP] system. With the exception of the initial Eu datum there is a relatively steady increase as the absorbed dose increases to $200 \mathrm{kGy}$, which would also be consistent with the lactate aqueous phase data shown for the same ligand in Fig. 4. If the phosphonic acid product of HEH[EHP] radiolysis is less water soluble than the phosphoric acid product of HDEHP radiolysis, this may explain the continued tendency toward increasing distribution ratios found upon replacement of the aqueous phase. Substitution of citric acid for lactic acid did not appear to change the behavior of the Advanced TALSPEAK system, although it must be cautioned that the citric acid-containing aqueous phase was not irradiated in these experiments. 


\section{RADIOLYSIS OF OTHER SOLVENT CONSTITUENTS}

In added scope, the radiolytic degradation of lactic acid and DTPA were also measured on biphasic, aerated irradiated samples. The aqueous phase of the irradiated biphasic samples contained $0.05 \mathrm{M}$ DTPA/1.5 M lactate and these species concentrations were measured in post-irradiation solutions. Shown in Fig. 6 are the results for the decrease in lactic acid concentration for irradiated contact with both the TALSPEAK and Advanced TALSPEAK organic solvents, as measured by ion chromatography. The decrease in concentration of lactic acid was linear with absorbed dose, and the slopes of the linear regressions are the $-G_{\text {lactic acid }}$ values in $\mu \mathrm{mol} \mathrm{Gy}{ }^{-1}$. The decomposition of lactic acid is fast in both systems, and probably similar, although there may be some scatter in the last two points on the Advanced TALSPEAK curve in Fig. 6.

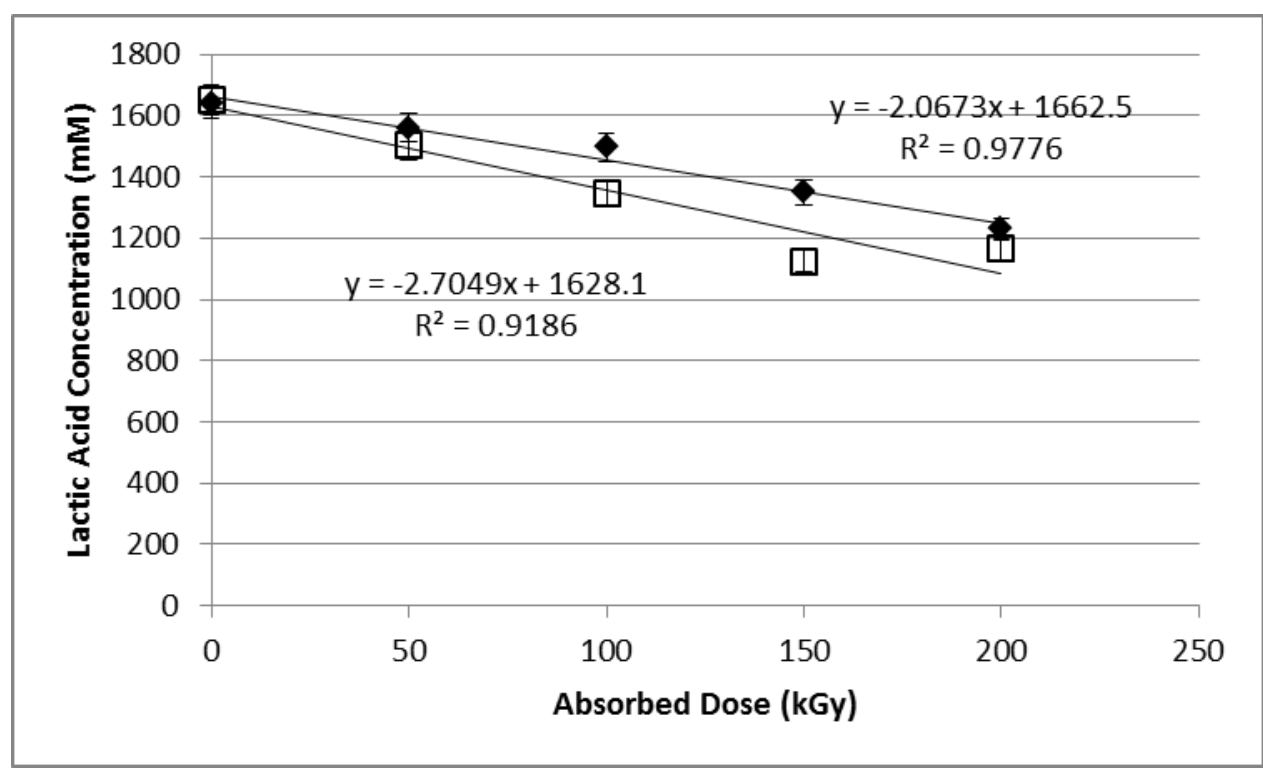

Figure 6. The radiolytic decomposition of aqueous lactic acid in the aerated, irradiated biphasic system with an organic phase composed of 0.5 M HDEHP/dodecane (closed diamonds: TALSPEAK) or $1.0 \mathrm{M}$ $\mathrm{HEH}[\mathrm{EHP}] /$ dodecane (open squares: Advanced TALSPEAK) . Error bars shown are $\pm 3 \%$ based on repetitive analyses.

The results for the change in DTPA concentration are shown in Fig. 7. Its concentration change was also linear with absorbed dose, and the slopes were similar in both systems, at a rate about $-\mathrm{G}_{\mathrm{DTPA}}=0.14 \mu \mathrm{mol}$ $\mathrm{Gy}^{-1}$. Thus, substitution of HEH[EHP] for HEDHP does not appear to have adverse effects on other system components. The decrease in DTPA concentration may also account for the rise in distribution ratios seen for the extractions involving the irradiated aqueous phase in Figs. 3 and 4. 


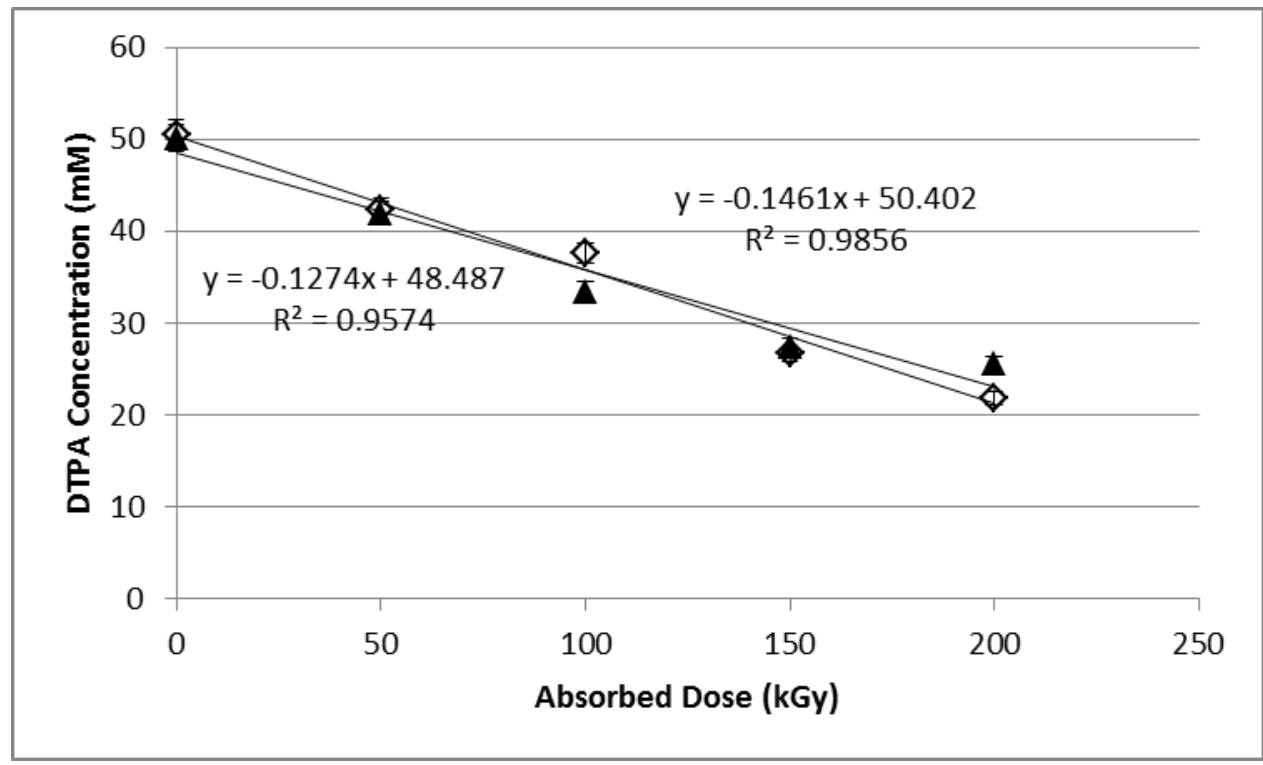

Figure 7. The radiolytic decomposition of aqueous DTPA in the aerated, irradiated biphasic system with an organic phase composed of $0.5 \mathrm{M}$ HDEHP/dodecane (open diamonds: TALSPEAK) or $1.0 \mathrm{M}$ HEH[EHP]/dodecane (closed triangles: Advanced TALSPEAK) . Error bars shown are $\pm 3 \%$ based on repetitive analyses.

\section{CONCLUSIONS AND FUTURE WORK}

Laboratory studies of the radiation chemistry of the TALSPEAK and Advanced TALSPEAK solvents were initiated with emphasis on the behavior of HEH[EHP] in the aerated biphasic system. These aerated biphasic conditions were chosen to best simulate potential process conditions. The degradation of $\mathrm{HEH}[\mathrm{EHP}]$ produced a product in common with that of HDEHP, probably $\mathrm{H}_{2} \mathrm{MEHP}$. A second product found only in Advanced TALPEAK samples may be the analogous phosphonic acid, both species produced by dealkylation. Future work will involve mass spectrometric confirmation of these proposed products.

The organic phases from the irradiated biphasic solutions above were used in solvent extraction experiments with Eu and Am-spiked aqueous phases appropriate to the TALSPEAK (DTPA/lactate for HDEHP) and Advanced TALSPEAK (HEDTA/citrate for HEH[EHP]) systems. The distribution ratios for both metals were flat to slightly increasing with absorbed dose in both systems, to the $200 \mathrm{kGy}$ maximum absorbed dose investigated. Additional solvent extraction experiments were conducted using the irradiated biphasic solutions. In this case the aqueous phase was the same (DTPA/lactate) for both organic phases since it was the aqueous phase present during the irradiations. Both systems showed either flat or slightly increasing $D_{\mathrm{Eu}}$ and $D_{\mathrm{Am}}$ versus absorbed dose under these conditions, however; separation factors did not appear to significantly change. The TALSPEAK and Advanced TALSPEAK systems behaved substantially similarly under irradiation. The degradation rate of the DTPA and lactate when in contact with either organic phase was also similar under irradiation.

Future work will include an irradiation of the optimized Advanced TALSPEAK process using the INL Irradiator Test Loop. The irradiation will be biphasic, and will use the HEH[EHP]/dodecane organic phase and the HEDTA/citrate aqueous phase. The effects of irradiation on distribution ratios, product formation and post-irradiation $\mathrm{pH}$ will be measured. 


\section{LITERATURE CITED}

1. Hudson, M. J.; Harwood, L. M.; Laventine, D. M.; Lewis, F. W. Use of Soft Heterocyclic N-Donor Ligands to Separate Actinides and Lanthanides. Inorg. Chem. 2013, 52, 3414-3428.

2. Todd, T. A. Separations Research for Advanced Nuclear Fuel Cycles. In Nuclear Energy and the Environment; Wai, C. M.; Mincher, B. J., Eds; American Chemical Society: Washington, DC, 2010; pp 13-18.

3. Horwitz, E. P.; Kalina, D. G.; Diamond, H.; Vandegrift, G. F.; Schulz, W. W. The TRUEX Process - A Process for the Extraction of the Transuranic Elements from Nitric Acid Wastes Utilizing Modified PUREX Solvent. Solvent Extr. Ion Exch. 1985, 3, 75-109.

4. Weaver, B.; Kappelmann, F. A. Preferential Extraction of Lanthanides Over Trivalent Actinides by Monoacidic Organophosphates from Carboxylic Acids and From Mixtures of Carboxylic Acids and Aminopolyacetic Acids. J. Inorg. Nucl. Chem. 1968, 30, 263-272.

5. Nilsson, M., Nash, K.L Review Article: A Review of the Development and Operational Characteristics of the TALSPEAK Process. Solvent Extr. Ion Exch. 2007, 25, 665-701.

6. Pereira, C.; Vandegrift, G. F.; Regalbuto, M. C.; Bakel, A.; Bowers, D.; Gelis, A. V.; Hebden, A. S.; Maggos, L. E.; Stepinski, D.; Tsai, Y.; Laidler, J. J. Lab-Scale Demonstration of the UREX+1a Process Using Spent Nuclear Fuel. Waste Management 2007, Tucson, AZ, February 25 - March 1, 2007; Tucson, AZ, 2007.

7. Braley, J. C.; Grimes, T. S.; Nash, K. L. Alternatives to HDEHP and DTPA for Simplified TALSPEAK Separations. Ind. Eng. Chem. Res. 2012, 51, 629-638.

8. Lumetta, G. J.; Neiner, D.; Sinkov, S. I.; Carter, J. C.; Braley, J. C.; Latesky, S. L.; Gelis, A. V.; Vandegrift, G. F. Combining Neutral and Acidic Extractants for Recovering Transuranic Elements from Nuclear Fuel. XIX International Solvent Extraction Conference, Santiago, Chile, Gecamin Ltda, Santiago, Chile: Santiago, Chile, 2011, Paper No. 68.

9. Lumetta, G. J.; Gelis, A. V.; Braley, J. C.; Carter, J. C.; Pittman, J. W.; Warner, M. G.; Vandegrift, G. F. The TRUSPEAK Concept: Combining CMPO and HDEHP for Separating Trivalent Lanthanides from the Transuranic Elements. Solvent Extr. Ion Exch. 2013, 31, 223-236.

10. Black, T. H. The preparation and reactions of diazomethane. Aldrichimica Acta 1983, 16, 3-10.

11. Tachimori, S. Radiolytic effects on the extraction of AmIII with di(2-ethylhexyl)phosphoric acid. J. Radioanal. Chem. 1979, 50, 133-142.

12. Tachimori, S. Synergistic extraction of americium with MEHPA DEHPA mixed solvent from nitric acid solution. J. Radioanal. Chem. 1979, 50, 143-151.

13. Draye, M., Favre-Réguillon, A., Chomel, R., Faure, R., Guy, A., Foos, J., Lemaire, M. Radiochemical stability of dicyclohexano-18-crown-6 ether (DCH18C6): synthesis and tests in radioactive medium of the DCH18C6 radiolytic products. Bull. Chem. Soc. Fr. 1996, 133, 183197. 
14. Kuruc, J. Spin trapping of free radicals generated by gamma radiolysis of crown ethers and their analogues. J. Radioanal. Nucl. Chem. Lett. 1990, 145, 205-213.

15. Zakurdaeva, O.A., Nesterov, S.V., Feldman, V.I. Radiolysis of aqueous DCH18C6 solutions at 77K. J. Radioanal. Chem. 2009, 279, 647-653.

16. Ershov, B.G., Seliverstov, A.F., Sukhov, N.L., Gordeev, A.V. Pulsed radiolysis of aqueous solutions of 18-crown-6. High Energy Chem. 1987, 21, 96-99.

17. Zakurdaeva, O.A., Nesterov, S.V., Feldman, V.I. Radical products of $\gamma$-radiolysis of 12 -crown-4 at 77K. High Energy Chem. 2007, 41, 65-70.

18. Mincher, B.J., Martin, L.R., Mezyk, S.P. Radiation chemistry in solvent extraction: FY2010 research. Report FCR\&D-SEPA-2010-0155.

19. Mincher, B.J., Modolo, G., Mezyk, S.P. Review article: the effects of radiation chemistry on solvent extraction: 1. Conditions in acidic solution and a review of TBP radiolysis. Solvent Extr. Ion Exch. 2009, 27, 1-25. 